\title{
Modeling and characterization of grain structures and defects in solidification
}

\author{
M. Rappaz \\ Laboratoire de Simulation des Matériaux, École Polytechnique Fédérale de Lausanne, 1015 Lausanne, Switzerland
}

\section{A R T I C L E I N F O}

Article history:

Received 27 March 2015

Revised 17 July 2015

Accepted 19 July 2015

Available online 26 July 2015

\section{Keywords:}

Grain structure modeling

Solidification defects

X-ray radiography

X-ray tomography

Orientation imaging

\begin{abstract}
A B S T R A C T
The paper by Karma and Tourret (this volume) in this special issue focuses on multiscale modeling approaches ranging from atoms to microstructure. In the present one, the most recent and significant modeling contributions dealing with the scale of solidification from microstructure to grain structure are briefly reviewed. The paper also covers modeling of defect formation during the last stage of solidification, namely porosity and hot tearing. As will be shown, the field of solidification has taken advantage of several simulation and experimental tools which have become increasingly powerful and accessible over the past decade. The emphasis will be put on complex 2D and 3D models for which correlations with in situ observations using synchrotron radiation and/or combined orientation and metallography imaging have been made.
\end{abstract}

(c) 2015 Elsevier Ltd. All rights reserved.

\section{Introduction}

Solidification has been one of the pioneering fields of materials science in which multiscale modeling aspects have been considered. One of the reasons is that phase transformations become simpler to approach when the assumption of a purely diffusion-controlled process is made (i.e., no convection in the liquid and no deformation of the solid for solidification). Under such assumption, solidification morphologies are controlled by thermal and solute diffusion and by the properties of the solid-liquid interface. Among those, the anisotropy of the solid-liquid interfacial energy, and to some extent that of the attachment kinetics, are key parameters. Grain boundary properties are also important for multi-phase solidification (eutectic, peritectic) The connection between the microstructure, grain structure and macroscopic scale of solidification processes has been a very active topic since the 1980 's [2-4]. With the arrival in solidification of the phase-field method in the mid 90's [5], it was initially thought that this technique would solve all the problems and cover all the scales, predicting microstructure and grain structure at the scale of an entire casting. Some attempts along this direction have been made, but at a huge computational cost on graphic processors and yet for a volume of a few tens of $\mathrm{mm}^{3}$ only [6]. After twenty years, we have to recognize that phase-field is "just" another, yet very powerful, tool to improve our understanding of solidification microstructures.

\footnotetext{
E-mail address: michel.rappaz@epfl.ch
}

It is now fairly well understood that a multiscale approach of solidification involves the prediction of the right properties at a given scale with an appropriate model. As input, it usually needs some information coming from a lower scale, while output results can be passed to a larger one. For example, as summarized in the paper of Karma and Tourret paper [1], the anisotropic properties of a solid-liquid interface can be deduced from atomistic simulations. These properties can then be used in phase-field calculations to predict dendrite growth orientation and kinetics. Such dendrite growth kinetics can then be used in micro-macro or mesoscopic models of solidification, i.e., models that make the connection between the microstructure and the process scale. Recent developments at this mesoscale have been made along two main directions. On one hand, analytical models of nucleation and growth, together with their coupling with heat-and-mass transfer calculations, have been refined. Their main goal is to improve predictions of macrosegregation and grain structures (columnar, equiaxed) in castings. On the other hand, similar goals have been set for so-called stochastic models of solidification such as CAFE (Cellular Automata coupled with Finite Elements). Compared to analytical models, this type of approach offers the additional advantage of providing a direct view of the grain structure and texture.

If models have made tremendous progress over the past two or three decades, analysis tools have also become more and more sophisticated. At present, one can have access to 3-dimensional (3D) information, sometimes also as a function of time, about the distribution, nature, composition and orientation of phases making 
a microstructure. This is notably the case of in situ X-ray radiography, diffraction, tomography or topography, thanks to high-power $\mathrm{X}$-ray beams delivered by dedicated synchrotron beam lines around the world. Crystallographic orientation of microstructures and grains are also now accessible in 2D sections thanks to automated orientation imaging technique such as Electron Back-Scattered Diffraction (EBSD). This can be extended to 3D if EBSD is combined with either Focused Ion Beam (FIB) or standard serial sectioning techniques. One has seen recently an increased number of publications combining sophisticated in situ observations with numerical simulations.

On the other hand, solidified microstructures are perhaps not as important as solidification defects (porosity, hot tearing) for the quality and mechanical properties of cast products. While there are many papers in the area of defect prediction, the present contribution will focus mainly on those where a link with microstructure and/or grain structure is made. In particular, one has seen recently an improved description of the last stage solidification of alloys. This is indeed the hot-cracking sensitive region of the mushy zone of dilute alloys. The gradual transition of the mushy zone leading to a continuous solid containing isolated liquid pockets (i.e., percolation of initially isolated grains) is now much better understood and analyzed.

The paper is subdivided as follows. Analytical micro-macro models of solidification are briefly reviewed in Section 2, while the main core of the paper (Section 3) is dedicated to stochastic models. Finally, the prediction of microporosity and hot tearing is described in the last section of the paper. In each section, the emphasis will be put on numerical simulations that are combined with in situ observations or experimental results.

\section{Analytical micro-macro models of solidification}

In the 1990's, C. Beckermann and his group derived the formalism to couple heat- and mass-transfer equations for a 2-phase solid-liquid system with microscopic model of solidification describing nucleation and growth of equiaxed grains $[7,3,4]$. Such micro-macro models are mainly used for the prediction of macrosegregation in large scale castings, including grain movement and sedimentation. In addition to macrosegregation maps, they provide access to spatial information about grain size distribution. Using the concept of grain envelope [8], three regions are distinguished in such models: the solid, the liquid within (intradendritic) and outside (extradendritic) the grain envelope.

These concepts have been used and refined in order to investigate the effect of thermosolutal natural convection, solidification shrinkage and motion of free floating grains on the resulting macrosegregation and grain density $[9,10]$. Assuming athermal nucleation on inoculant particles such as TiC characterized by a size distribution [11], the evolution of each class of inoculant particles can be tracked. These inoculant particles are assumed to be transported with the velocity of the liquid until they become active at their corresponding undercooling and become grains. Grains then move with the velocity of the solid phase. It is shown that different classes can be activated depending on the hypotheses. In the presence of grain motion, grains are nucleated in a small zone close to the cooled surface of the mold only. Some of them settle and pack at the bottom, while others travel through a low-undercooling zone before packing. Such simulations can possibly explain the duplex grain structure often observed in the center of ingots.

In $[12,13]$, the concept of a splitting operator introduced already by several authors, e.g. [14], is generalized and justified. The basic idea is to consider the different time scales that govern microscopic phenomena of grain nucleation and growth on one hand, and macroscopic phenomena of heat- and mass-transfer, including grain motion, on the other hand. An interesting benchmark for the simulation of macrosegregation and freckling in columnar grain structures (no grain movement) has been reported in [15]. Besides comparison with carefully calibrated experiments $[16,17]$, such benchmarks of complex codes are really necessary. Indeed, very small differences in the resolution scheme, method or mesh size can lead to slightly different results, especially for freckles.

Micro-macro models have also been used for the modeling of small Al-Cu droplet solidification [18] in order to predict the distribution of copper and to analyze the influence of the nucleation undercooling. They have also been extended to include the mold filling stage [19]. The influence of the equiaxed grain morphology (globular or dendritic) and of their transport on macro- and mesosegregation (freckles) has been demonstrated for large steel ingots in [20]. In such ingots, both columnar and equiaxed grain structures [21] have been accounted for using a micro-macro approach based on 5 "phases" [22,23]. This terminology is unfortunate in materials science since these are not phases, but regions or zones. They correspond in fact to: (i) the solid dendrite and (ii) interdendritic melt of equiaxed grain envelopes; (iii) the solid dendrites and (iv) interdendritic melt of columnar dendrite envelopes; and (v) the extradendritic melt. Each of these "phases" is characterized by a volume fraction and a velocity.

One of the weak points of such micro-macro modeling approaches is the transition of equiaxed grain morphologies. For small radius, equiaxed grains remain globular with the growth kinetics of a sphere. For large radius, they are fully dendritic and the growth kinetics of their envelope is essentially that of an isolated dendrite tip (until solute layers of neighbor grains start overlapping, i.e., "soft impingement"). The problem comes for intermediate growth radii when globular equiaxed grains become globular-dendritic, i.e., the equiaxed equivalent of cells in directional solidification: For such morphologies, there is no analytical solution for the growth kinetics. In this respect, phase-field simulations such as reported in [24], or a simple globular-dendritic transition (GDT) criterion, such as the one derived in [25], can help improve the description of this transition and thus micro-macro models.

\section{Stochastic grain structure models}

The Cellular Automata coupled with Finite Elements (so-called CAFE model) [26,27] was developed in the 1990's. Compared to analytical micro-macro models of solidification, it offers the advantage of providing a direct view of the grain structure (columnar and equiaxed) and of the texture. Such model has since been improved in many ways by many authors, but in particular by the group of Gandin at CEMEF. From a computational point of view, the dynamic allocation of the memory already implemented in the original CAFE approach to track the liquidus [27] has been improved. Several new algorithms and parallelization strategies have been implemented [28] in order to predict grain structure formation for much larger castings within reasonable computation times. In [28], an ingot of about $0.2 \mathrm{~m}^{3}$ containing more than 1 million grains has been simulated with CAFE and about $4.9 \cdot 10^{9}$ cells.

Such computational optimisations are also necessary if one wants to couple CA with average equations of mass, momentum and solute in order to predict convection and macrosegregation. A very good example of such a CAFE calculation combining grain growth, thermosolutal convection and macrosegregation is given in [17]. These authors have simulated the solidification of a Sn $3 \mathrm{wt} \% \mathrm{~Pb}$ alloy in a rectangular cavity having the dimensions of Hebditch and Hunt's experiment [29]. However, the experiment 
has been improved to control the heat exchange of this cavity with the surrounding. The temperature of the smallest opposite faces are totally controlled and the thermal losses of the four others are compensated by a Kirchhoff box to have adiabatic conditions. Further details of this benchmark experiment can be found in [16]. A result of such simulations is shown in Fig. 1. As can be seen, solutal convection dominates in this experiment, and this influences the growth of columnar tin grains and the columnar-to-equiaxed (CET) transition.

Another interesting extension and application of the CAFE model is shown in Fig. 2 [30,31]. It shows a comparison with in situ X-ray radiography solidification experiments performed on the ID19 beamline of the ESRF synchrotron facilty. A thin Al-3.5 wt.\% Ni specimen inoculated with 0.5 wt.\% AlTiB was directionally solidified and observed in situ. After $4200 \mathrm{~s}$, the pulling velocity of the specimen was increased from 1.5 to $4 \mu \mathrm{m} / \mathrm{s}$. This increased the undercooling of the columnar front, thus allowing the formation of a few equiaxed grains ahead of the columnar front (left image in Fig. 2), i.e., start of a CET. In this configuration, the thermal gradient is vertical and the heavier nickel has a segregation coefficient smaller than 1 . Therefore, the gradient of liquid density is expected to be parallel to gravity and there should be no convection. However, as can be seen, the columnar dendrite front is not horizontal in Fig. 2. This indicates that the isotherms are not precisely horizontal, thus initiating a flow of liquid towards the edges of the thin specimen with a positive macrosegregation at those locations [25]. The position and orientation of each grain were then fed to the $2 \mathrm{D}$ version of the CAFE model, coupled with
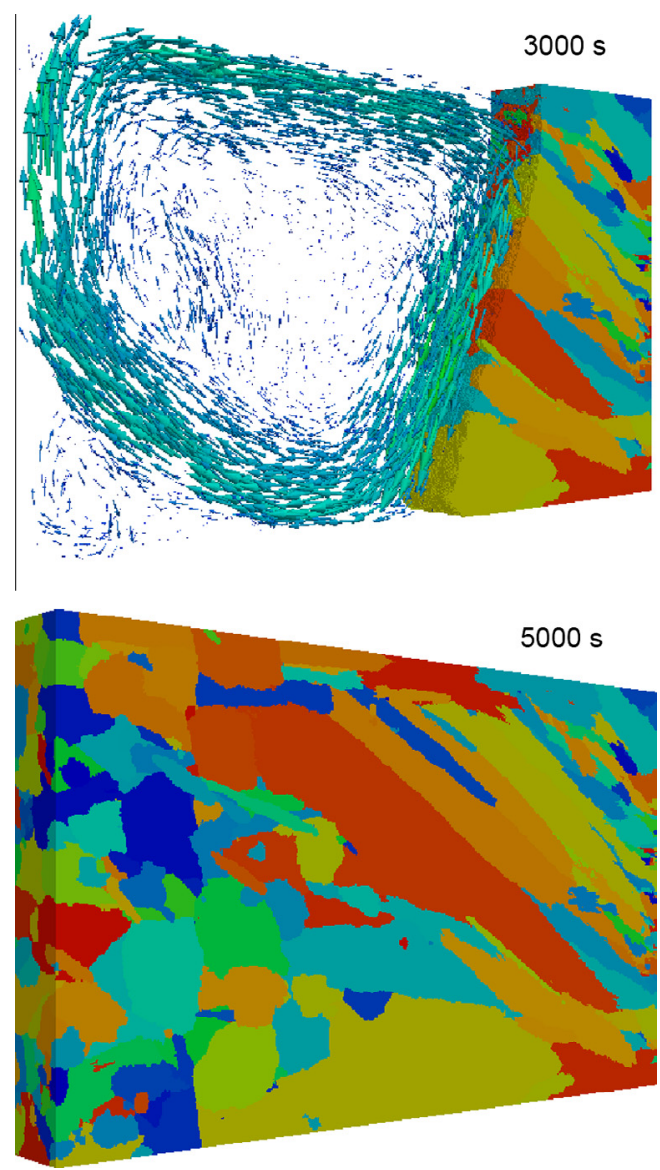

Fig. 1. Grain structure and velocity field at two instants in a $\mathrm{Sn}-3 \mathrm{wt} . \% \mathrm{~Pb}$ alloy solidified in a rectangular cavity $10 \times 6 \times 1 \mathrm{~cm}^{3}$ which has temperature-controlled external surfaces [17].
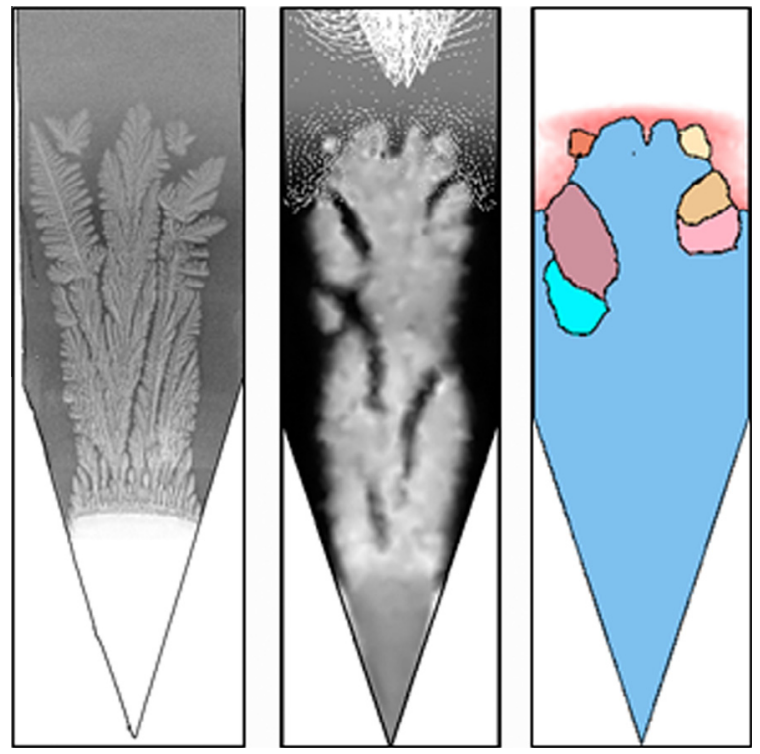

Fig. 2. In situ X-ray radiography of an Al-3.5 wt.\% Ni specimen (left) showing the columnar dendritic front with a few equiaxed grains forming just ahead at the time the pulling velocity of the specimen was changed from 1.5 to $4 \mu \mathrm{m} / \mathrm{s}$. The computed grain structure and concentration field calculated with CAFE are displayed on the right and center, respectively [30].

fluid flow and macrosegregation calculations. The figure at the center of Fig. 2 shows the calculated Ni composition profile, with the enrichment near the edges. The grain structure map on the right clearly reproduces the shape of the curved columnar front.

Although the extension and recent applications of CAFE are quite impressive, it does not mean that some of its assumptions cannot be put into question. Since the grains are assumed to be dendritic with the growth kinetics taken from that of an isolated tip, the model does not apply to globular or globular-dendritic equiaxed grains (see previous remark for analytical models). One of the basic principles of the original CAFE model [26] is that dendrites are constrained to grow along $\langle 100\rangle$ directions. As such, it is not directly applicable to other situations, such as hexagonal-type elements (e.g., $\mathrm{Mg}$ or $\mathrm{Zn}$ ) or fcc metals having other growth directions [32]. The grains in CAFE also interact via "hard-impinge ment", i.e., they do not slow down when their surrounding solute layers overlap. This has some implication on the competition between columnar grains. Columnar $\langle 100\rangle$ dendrites well aligned with the thermal gradient have a lower growth velocity, and thus a smaller undercooling, than those of a misaligned grain. At a converging grain boundary, misaligned dendrites are thus slightly behind those of the well-aligned grain and the boundary is locked. However, Zhou et al. [33] have shown in a bicrystal Ni-base superalloy that such is not strictly the case. The solutal interaction between two converging neighboring dendrite tips slows them both down. This allows the next misaligned dendrite tip neighbor to grow ahead and bypass the well-aligned dendrite located at the grain boundary. As a consequence, the converging grain boundary moves by one dendrite trunk spacing in the well-aligned grain when "soft" grain impingement (i.e., solute interactions) is considered. Such a phenomenon, which is not accounted for in the standard CAFE approach, has been modeled in 2D with the phase-field method [34]. But to be realistic, dendrite growth competition at grain boundaries should be calculated with phase field in $3 \mathrm{D}$, as in [6], in order to improve the growth algorithm of CA's. An alternative solution, with lower computation costs, could be provided by the Dendrite Needle Network (DNN) developed by Tourret and Karma and already described in [1]. 
Since the beginning of 2000 , one has seen the emergence of so-called "modified CAFE" models. These models solve the solute diffusion equation and thus can handle "soft" grain impingement. They estimate the curvature of the solid-liquid interface on the basis of the solid fraction of neighbor cells $[35,36]$ (see also the fairly complete reference list in [37]). Besides "soft" impingement, such modified CA have the advantage over simpler CA that they produce dendritic patterns within the grains that look like those produced by phase field calculations. They can mimic the dendrite competition that occurs among different dendrite variants in a single crystal [37], the selection of primary trunks within a grain [35] and the dendrite competition at grain boundaries. They can also be coupled with heat- and mass-transfer equations for the prediction of macrosegregation and freckles formation, as illustrated in Fig. 3. In the experiment of Shevchenko et al. [38], a thin Ga-25 wt.\% In specimen is solidified directionally while X-ray radiographies are recorded. The gray-level contrast pictures are then converted into a color code that reflects the composition. In this system, indium dendrites solidify first, thus rejecting lighter gallium in the liquid. This leads to the formation of solute plumes and freckles, which strongly modify the growth of columnar grains. They can also fragment dendrite arms which then become equiaxed grains falling back on top of the columnar front or in the freckles. Such a situation has been modeled in [39] using the code $\mu$ MatIC, which is based on the modified CA of Wang et al. [35].

Although modified CA overcome some limitations of CAFE and produce phase field-like dendritic patterns, the underlying hypotheses and limitations should not be forgotten. In particular, the growth algorithm cannot predict correctly the actual kinetics of dendrite tips. The dendrite tip kinetics has almost never been validated on analytical solutions or phase field computations. Furthermore, the dendrite growth directions are biased by the mesh unless a huge anisotropy of the solid-liquid interfacial energy, much larger than the few percents of real metallic alloys, is introduced. In order to be close to the phase-field method accuracy, modified CA require a much finer mesh and a much more refined algorithm for the estimation of interfacial curvature. This is precisely what is done in the level set [40] or pseudo-front tracking [41] methods. But these methods have a computation cost

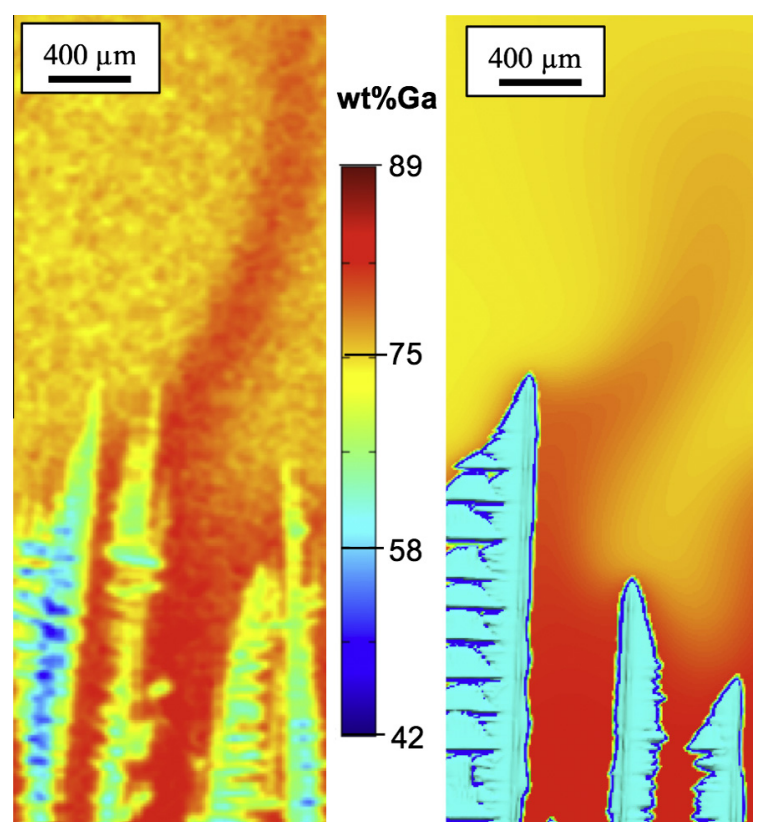

Fig. 3. In situ X-ray radiography of a Ga-25 wt.\% In alloy (left) showing the formation of freckles and corresponding simulation with $\mu$ MatIC (right) [39]. comparable with that of the phase-field method, while they are more difficult to extend to 3D.

Another mesoscale approach to compute the formation of grains is the so-called "mesoscopic" model initially developed for pure substances by Steinbach et al. [42], and later adapted to alloys in [43]. It accounts for solutal interactions between grains without providing a resolution of individual dendrite arms. A confocal envelope of a grain, i.e., a surface located at a fixed distance from that surrounding the "active" dendrite arms, is first defined. The velocity of the dendrite tips and "active" arms is provided by an analytical solution, as in the regular CAFE model, but with the undercooling across a stagnant film surrounding the envelope. From its construction, it handles cruciform envelope grain shapes, as opposed to the regular CAFE model which considers octahedra. It also considers "soft" grain impingement. Recently, such a model has been applied to analyze in situ X-ray radiographies of $\mathrm{Al}$ $30 \mathrm{wt} . \% \mathrm{Cu}$ alloys [44]. The in situ observations and mesoscopic simulations shown in Fig. 4 illustrate how a primary dendrite trunk is eliminated during growth by solute rejected by too-closely packed dendrite trunk neighbors.

\section{Modeling of microporosity and hot tearing}

Shrinkage microporosity and hot tearing typically occur in the last stage solidification of low-concentration alloys. Since they are the main limiting factors for the mechanical properties of cast products, in particular ductility, toughness and fatigue strength, one has seen a regain of interest to model their formation with more accurate simulation tools. In the same way, in situ observation techniques have allowed them to be visualized in real alloys during solidification. We will restrict our discussion to those models and approaches in which a clear link with the solidification microstructure is made.

\subsection{Microporosity}

Numerical models of microporosity formation have been pioneered by Piwonka and Flemings [45] and later refined by Kubo and Pehlke [46]. As reviewed in [47] or explained in basic textbooks [25], these models first solve the mass- and momentum conservation equations in the mushy zone, with appropriate boundary conditions. Assuming a Darcy-type liquid flow in between fixed dendrites, this allows to calculate the pressure drop in the liquid of the mushy zone. Under simplified assumptions and 1D configuration, analytical expressions can even be calculated [25]. Things get more complicated when microporosity models consider the formation of the new phase, i.e. the pores. Indeed, nucleation and growth of pores require to account for segregation and diffusion of gaseous and/or volatile solute elements into the liquid. Accordingly, the supersaturation of the melt associated with such elements has to be considered. It is a function of the pressure, besides temperature and composition, of the liquid. Furthermore, the formation of voids partially compensates solidification shrinkage, and thus relaxes the local liquid pressure.

A fairly general formalism to encompass multiple dissolved gas elements, such as $\mathrm{H}_{2}$ or $\mathrm{N}_{2}$, as well as volatile solute elements, such as $\mathrm{Zn}$, in the equilibrium conditions has been made in [48]. The effect of limited hydrogen diffusion in aluminum alloys on the pore fraction has been evidenced in [49], while the diffusion coefficient of this element in a two-phase solid-liquid mixture has been calculated in [50]. But the most important refinements of microporosity models are related to curvature of micropores. Since shrinkage micropores have to grow in between well-developed dendrites (or grains), they have a very complex and tortuous morphology, as shown in Fig. 5 [51]. 

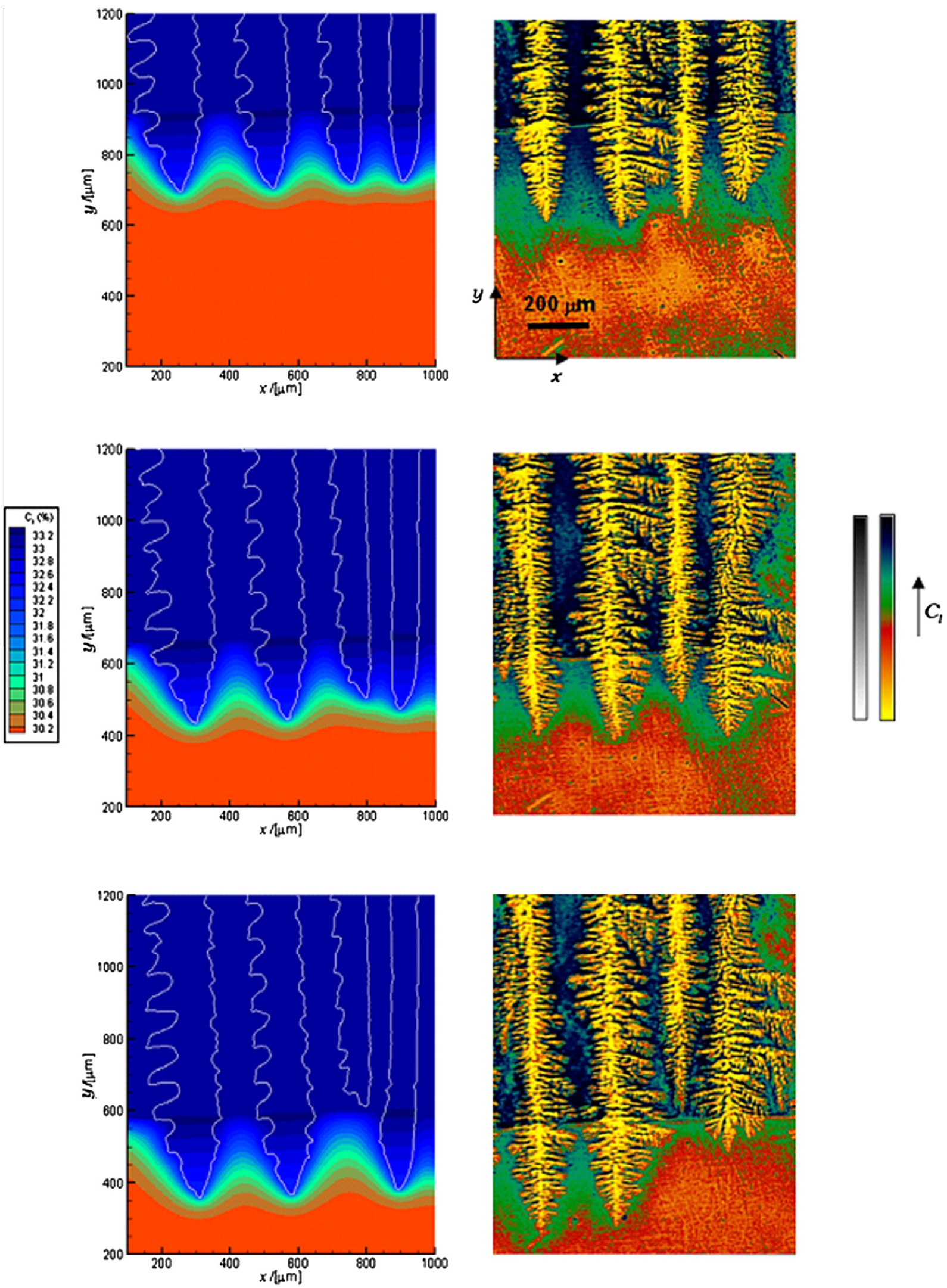

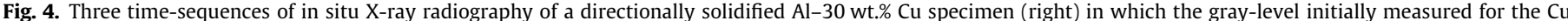

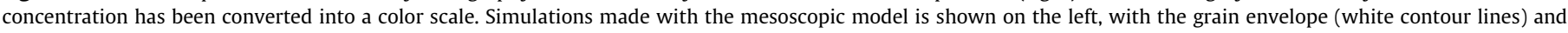
composition field (in color) [44]. 

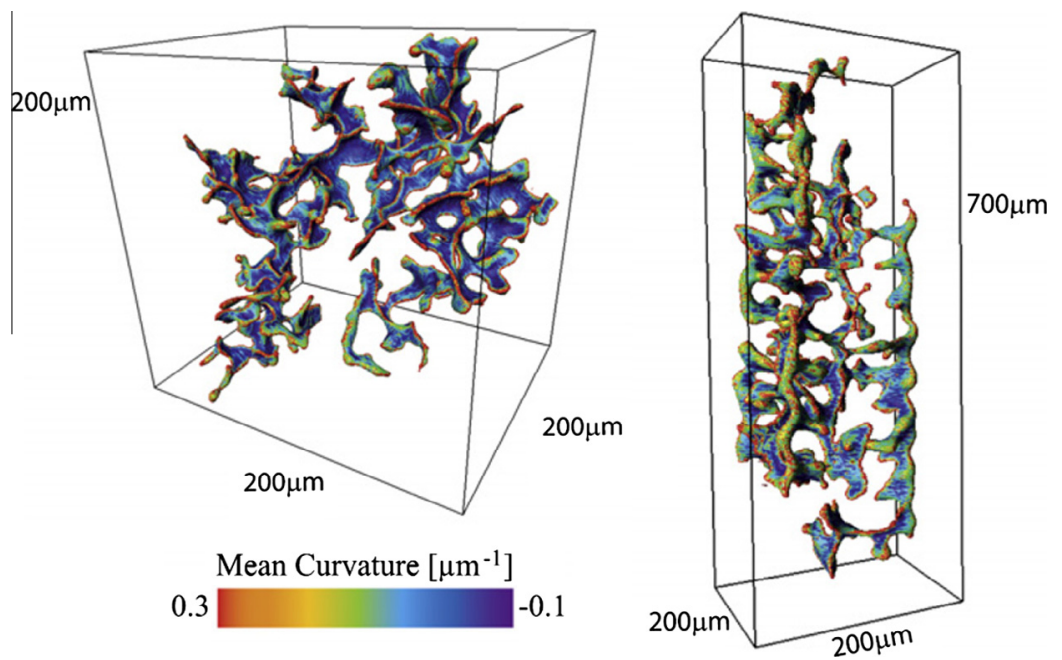

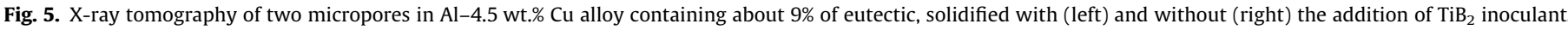
The surface of the pores has been colored as a function of the local mean curvature [51].

This 3D X-ray microtomography reconstruction reveals shrinkage mircopores in $\mathrm{Al}-\mathrm{Cu}$ alloys. When the alloy is inoculated with $\mathrm{TiB}_{2}$ particles, the grains are equiaxed and the overall morphology of micropores is also fairly "equiaxed" (Fig. 5, left). Without inoculation, the primary dendrites are columnar and micropores adopt an elongated shape surrounding the primary trunks (Fig. 5, right). The "negative" shape of columnar dendrites, with primary and secondary arm spacings, is clearly visible in the second microtomograph. The 3D shape of the micropores being reconstructed, the two local principal radii of curvatures of its surface can be calculated in order to construct an Interface Shape Distribution (ISD) map. Such maps were originally proposed by Voorhees and his group for the study of coarsening in alloys [52]. The ISD plots corresponding to Fig. 5 indicate that most of the surface of the micropores has a saddle-type morphology. The smallest principal curvature is negative (average of about $-0.1 \mu \mathrm{m}^{-1}$ ), while the other one is positive. The associated negative radius of curvature $(-10 \mu \mathrm{m})$ corresponds roughly (with the opposite sign) to half the secondary dendrite arm spacing $\left(\lambda_{2}=20 \mu \mathrm{m}\right.$ in this case). Such saddle-type shape regions correspond to the surface of the pore in contact with the solid dendrites.

The micropore surfaces in Fig. 5 have been colored according to the local mean curvature. As can be seen, most of the surface has a slightly negative mean curvature (blue-green regions, -0.1 to $0 \mu \mathrm{m}^{-1}$ ) and corresponds to the micropore-primary dendrite interface. A few regions have a very high positive mean curvature (red regions, $0.3 \mu \mathrm{m}^{-1}$ ): They correspond to the regions of the surface of the micropore in contact with the interdendritic regions, i.e., the eutectic. It is not sure that the micropore had the same mean curvature when it was in contact with the last interdendritic liquid of eutectic composition, just before it solidified. But if we make this assumption, the capillary overpressure associated with a curvature of $0.3 \mu^{-1}$ correspond to a capillary pressure of about $600 \mathrm{kPa}$ (the liquid-air interfacial energy is about $1 \mathrm{~J} / \mathrm{m}^{2}$ for $\mathrm{Al}$ ). This contribution is very often larger than the pressure drop in the liquid and can compensate the negative pressure reached sometimes in the interdendritic liquid, deep in the mushy zone.

In order to estimate more accurately this capillary overpressure during solidification, the multiphase-field method has been used recently to simulate the growth of a pore in simple configurations [53] or in a representative volume element extracted from tomography measurements [54]. The group of Lee et al. implemented the same concepts in a modified CA to predict and visualize microporosity formation in $\mathrm{Al}-\mathrm{Cu}-\mathrm{Si}$ alloys [55].

\subsection{Hot tearing}

The problem of hot tearing has seen a regain of interest and new approaches over the past fifteen years. Simple models such as the RDG criterion [56], similar to the Niyama criterion for microporosity formation [25], or more complex models based on averaged conservation equations $[57,58]$ have been developed. On the other hand, the rheological behavior of alloys in the semi-solid state has been measured (e.g. [59] for Al alloys). It is well-known that hot tearing is an intergranular defect that forms as a result of thermal strains in the coherent solid and insufficient liquid feeding. Its intergranular nature is linked with the presence of thin liquid films that remain at grain boundaries of dilute alloys until a late stage of solidification. These films cannot sustain mechanical tensile and shear strains induced and transmitted by the coherent solid and thus behave as a brittle phase. Consequently, localized strains at those "wet" grain boundaries can no longer be compensated by liquid feeding due to the very low permeability of the mushy zone.

Average approaches such as [56-58] are definitely useful for the prediction of hot cracking tendency of alloys in solidification processes such as continuous casting or welding. However, refined models which account for the grain structure are needed if the intergranular and localized nature of hot tears is to be described. For that purpose, the coalescence of two solid-liquid interfaces leading to the formation of a grain boundary had to be described first. Using a simple thermodynamic approach, it was shown in [60] that two types of grain boundaries have to be distinguished: (i) when the grain boundary energy $\gamma_{g b}$ is smaller than $2 \gamma_{s \ell}$, where $\gamma_{s \ell}$ is the solid-liquid interfacial energy, the boundary is "attractive". The two solid-liquid interfaces tend to immediately coalesce when they get within interaction distance; (ii) when $\gamma_{g b}>2 \gamma_{s \ell}$, the boundary is repulsive. A so-called coalescence or bridging undercooling is required to overcome the surface energy barrier given by $\left(\gamma_{g b}-2 \gamma_{s \ell}\right)$. Molecular dynamic simulations with an embedded atom approximation have shown a slightly more complex behavior for pure nickel [61]. A symmetric $\Sigma 9$ twist boundary shows a fairly simple repulsive behavior, but a symmetric $\Sigma 9$ tilt boundary exhibits a more complex behavior: it has a weak attractive disjoining 
potential at larger boundary width and a repulsive behavior at short width. The influence of the misorientation angle of a symmetric tilt boundary on the hot tearing sensitivity of a laser weld of a Ni-base superalloy bicrystal was clearly evidenced in [62].

On the basis of such coalescence concepts, the percolation of a population of randomly oriented equiaxed-globular grains has been investigated, in 2D [63] and 3D [64]. Percolation is essential for hot tearing since it determines when the coherent solid can transmit tensile stresses across a domain. Using a Voronoï tessellation of randomly distributed grains, solidification was first calculated. The result was then used to calculate fluid flow within the intergranular liquid channels, using a Poiseuille-type approximation. This fluid flow model was coupled with the movement of rigid grains in 2D [65] or with 3D thermomechanical calculations for the solid phase [66]. Finally, the combined fluid flow - deformation calculation was completed with a cracking-criterion based on cavitation in the intergranular liquid (see below).

In parallel to these "granular" model developments, in situ tensile tests were performed by Terzi et al. on the X-ray microscopic tomography beam line of ESRF [67]. Fig. 6, left, shows a longitudinal section of an $\mathrm{Al}-8 \mathrm{wt} . \% \mathrm{Cu}$ specimen strained at $555^{\circ} \mathrm{C}$ (just above the eutectic temperature) and at a speed of $0.1 \mu \mathrm{m} / \mathrm{s}$. The liquid of eutectic composition is white in this reverse contrast tomogram, while solid $\mathrm{Al}$ and voids are shown in gray and black, respectively. The specimen has been machined with a notch prior to the in situ tensile test in order to localize the strains in the

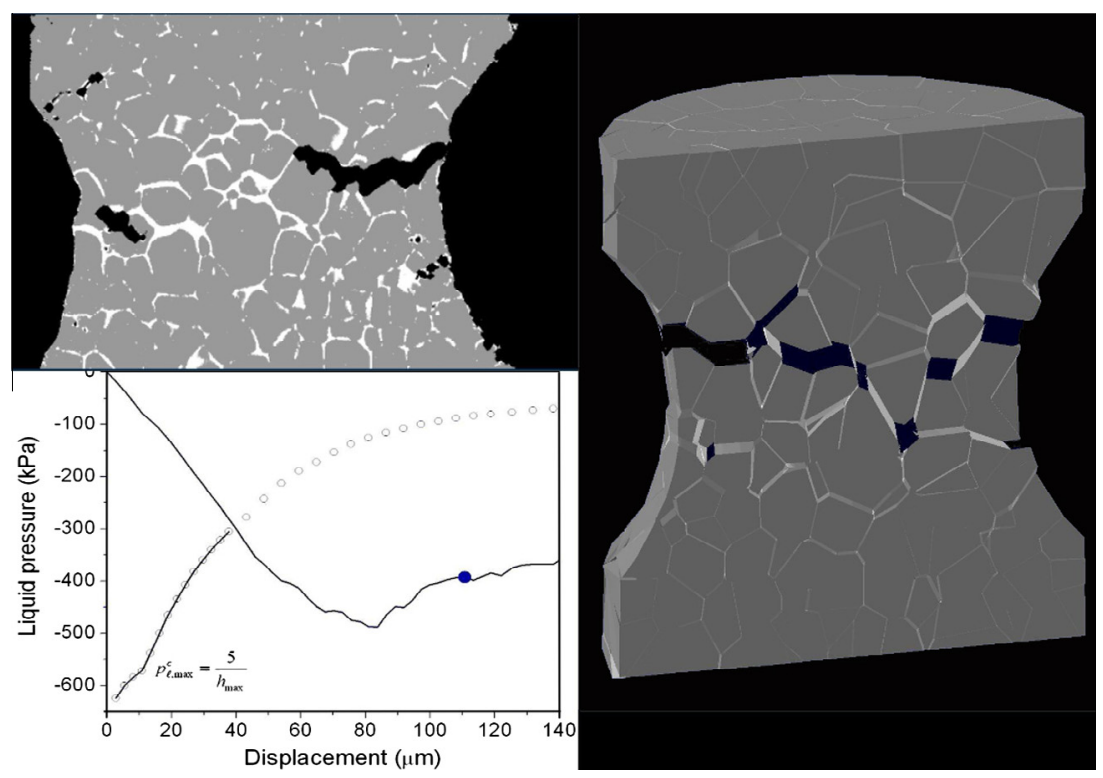

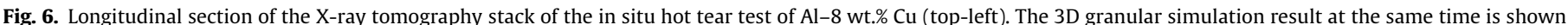

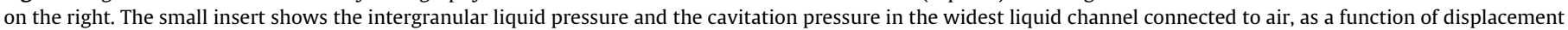
$[67,68]$.
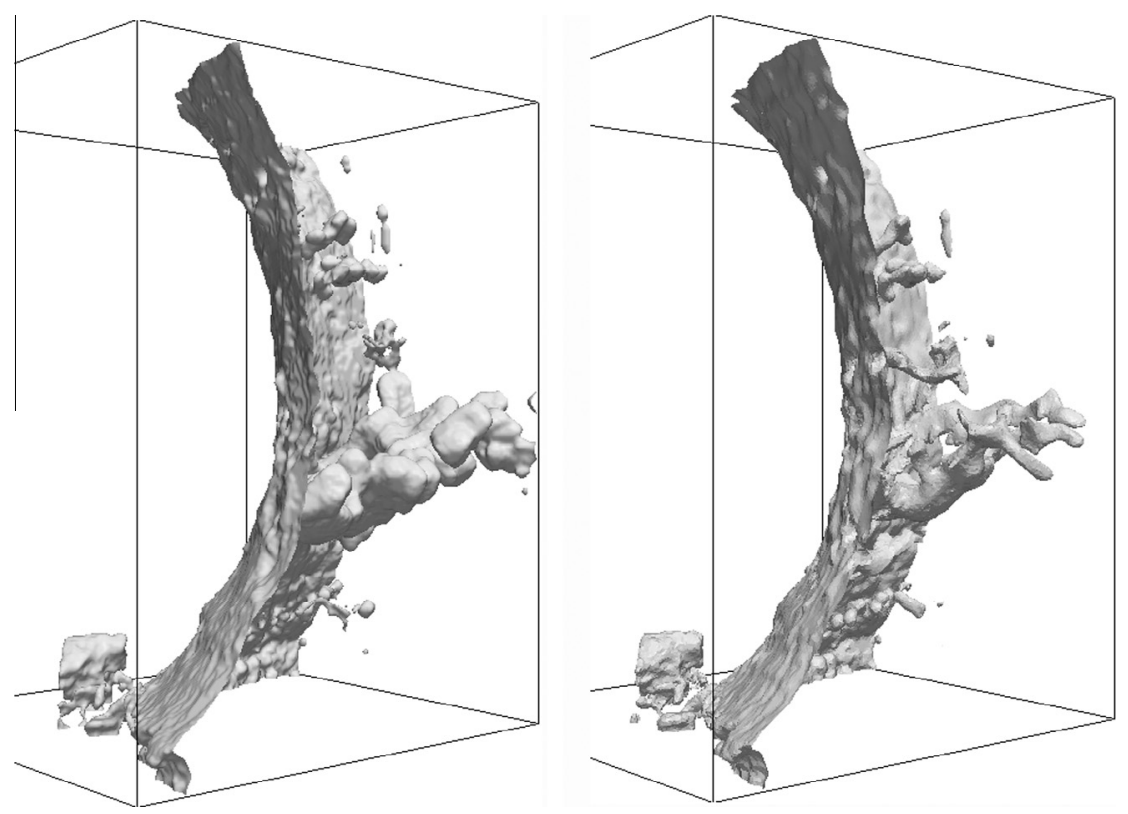

Fig. 7. X-ray tomography (left) and level-set simulation (right) of Al-8 wt.\% Cu [69]. 
observation zone. In a first stage of deformation, grains deform and rearrange so as to accommodate the strain. During this stage, the intergranular liquid is drained in the highly strained region (the notch) from the upper and lower parts of the specimen. This can be measured directly from the fraction of liquid: uniform with about $7 \%$ before deformation, it increases in the notch region during deformation. Once this mechanism is no longer possible, cracks are initiated. Time-resolved X-ray tomography has shown that they form at the periphery of the specimen, where the new phase (air) is already available (no crack nucleation necessary in this case). They tend to form in the widest liquid channels since the air-liquid meniscus has the lowest curvature (and thus capillary overpressure) in such regions.

The same situation has been simulated with the granular model [68], as shown on the right of Fig. 6. Although grain boundaries are flat surfaces in this Voronoï model, the same mechanisms are fairly well reproduced. The small graph shows two curves as a function of the displacement of the jaw of the micro-tensile test: The calculated intergranular liquid pressure (curve going down) and the calculated capillary pressure associated with the liquid-air (in fact oxide skin-air) meniscus in the widest liquid channel connected to the exterior (curve going up). When these two curves cross, the first void is initiated at the periphery of the specimen in the widest channel and then propagates inside.

Another modeling approach to this tensile test has been made in [69] using a level set method. The initial configuration of solid grains, intergranular liquid and air has been taken first from the $\mathrm{X}$-ray tomography stack. The 3-phase system was then simulated assuming an elastic behavior with different elastic modulus. Fig. 7 shows a comparison of the calculated and observed deformed specimen after some stage of deformation. Although the mechanical behaviors of liquid and air are poorly approximated, the model reproduces remarkably well the introduction of air (crack) within the notched area.

\section{Conclusion}

Modeling of solidification grain structures and defects has made significant progress over the past five to ten years. Besides cheaper and more powerful computers, improvements of the models have been made possible thanks to new or optimized numerical methods. As importantly, our understanding of the key phenomena has improved with the help of careful experiments and simulation. In this respect, simulation is a wonderful "learning" tool, probably as important as a fitting method. This short paper has reviewed some of these developments and is by far not exhaustive. The emphasis has been put on contributions in which a clear link with the microstructure is made.

For large castings, analytical micro-macro models of solidification are still very valuable for the prediction of macrosegregation. The transport of inoculant particles and grains very often plays a significant contribution. For smaller castings (e.g., turbine blades), the continuous improvement of Cellular Automata has shown its potential to incorporate many phenomena, in particular convection and macrosegregation. The criteria of grain propagation in the CA should be refined in order to include solutal interactions between dendrite tips ("soft" grain impingement). This is particularly important at convergent grain boundaries. From that point of view, modified CA, DNN or mesoscopic models of solidification have an advantage. When trying to include a curvature contribution in so-called modified CA to produce dendrite-like patterns, it should be kept in mind that the shape, growth direction and kinetics of dendrites simulated with such techniques are qualitative only.

For the prediction of microporosity, especially when it occurs at a late stage in solidification, the capillary overpressure plays a major role and has been too often underestimated in the models. This overpressure directly influences the fraction of pores since this phase is compressible. Considering the importance of this defect for the mechanical properties of cast components, further work is needed and not only on capillary effects. As shown by in situ X-ray tomography, shrinkage porosity appears quite suddenly when the interdendritic eutectic solidifies. Under such conditions, it is not sure that local thermodynamic equilibrium (including curvature) applies.

Substantial progress has been made in the area of hot tearing prediction. The development of average 2-phase methods accounting for the very different behaviors and interaction of the solid and liquid is an important step to predict this defect in industrial castings and in welding. At a more microscopic scale, granular models and in situ hot tearing tests have enlightened the basic mechanisms. The two approaches could probably be combined in the future. Average 2-phase models can be used in a first step to identify critical regions of the casting/welding where hot tears are mostly susceptible to form. They also provide boundary conditions to be applied in such critical zones for a finer microscopic simulation at the scale of the grains. Such types of approaches have already been used for crack propagation in solids.

Finally, it is important to recall that reliable simulations require accurate thermophysical data. Although several are fairly well known (e.g., thermal conductivity, latent heat of fusion, phase diagram, ...), many need to be more accurately assessed, in particular diffusion coefficients and properties related to solid-liquid and solid-solid interfaces. Their temperature- and/or solute dependence is very often ignored. Yet, they strongly influence microstructure characteristics such as growth directions and kinetics, and thus grain structures. Carefully controlled experiments and atomistic simulations based on accurate potentials are really needed in this area to improve phase field simulations and the lower scale of mesoscopic models. On the upper scale of such models, efforts should be made to make those simulations useable in industrial applications. Finally, if growth kinetics models are fairly well established, phenomena related to grain "birth" need to be studied in greater depth, sometimes with "new eyes". Production of new grains by dendrite fragmentation should consider remelting, for which there are limited studies only [70]. Heterogeneous nucleation on foreign particles such as $\mathrm{Al}$ on $\mathrm{TiB}_{2}$ or $\mathrm{TiC}$ particles has been shown to be athermal and thus does not follow standard theories, at least at small undercooling [11]. Finally, it has been shown recently that homogeneous nucleation in the liquid might depend on the short range order of atoms in the liquid, itself influenced by minute additions of solute elements [71].

\section{References}

[1] A. Karma, D. Tourret, COSSMS, this volume, 2015.

[2] M. Rappaz, Int. Mater. Rev. 34 (1989) 93.

[3] C. Wang, C. Beckermann, Met. Mater. Trans. A 27 (1996) 2754.

[4] C. Wang, C. Beckermann, Met. Mater. Trans. A 27 (1996) 2765.

[5] J. Warren, W.J. Boettinger, Acta Metall. Mater. 43 (1995) 689.

[6] T. Takaki, T. Shimokawabe, M. Ohno, A. Yamanaka, T. Aoki, J. Cryst. Growth 382 (2013) 2125.

[7] J. Ni, C. Beckermann, Met. Mater. Trans. B B22 (1991) 349.

[8] M. Rappaz, P. Thévoz, Acta Metal. 35 (1987) 1487.

[9] M. Bedel, K.O. Tveito, M. Založnik, H. Combeau, M. MHamdi, Comput. Mater. Sci. 102 (2015) 95109.

[10] M. Založnik, A. Kumar, H. Combeau, M. Bedel, P. Jarry, E. Waz, in: J.F. Grandfield, D.G. Eskin (Eds.), Cast Shop for Aluminum Production, Essential Readings in Light Metals, vol. 3, John Wiley \& Sons, 2013, pp. 848-853.

[11] A.L. Greer, P.S. Cooper, M. Meredith, W. Schneider, P. Schumacher, J.A. Spittle A. Tronche, Adv. Eng. Mater. 5 (2003) 81.

[12] M. Založnik, H. Combeau, Comput. Mater. Sci. 48 (2010) 1.

[13] M. Založnik, A. Kumar, H. Combeau, Comput. Mater. Sci. 48 (2010) 11.

[14] J. Guo, C. Beckermann, Numer. Heat Trans. A A44 (2003) 559.

[15] H. Combeau, M. Bellet, Y. Fautrelle, D. Gobin, E. Arquis, O. Budenkova, B. Dussoubs, Y.D. Terrai, A. Kumar, C. Gandin, B. Goyeau, S. Mosbah, T. Quatravaux, M. Rady, M. Založnik, in: A. Ludwig (Ed.), MCWASP XIII: 
International Conference on Modeling of Casting, Welding and Advanced Solidification Processes, IOP Conference Series, vol. 33, 2012.

[16] L. Hachani, B. Saadi, X.D. Wang, A. Nouri, K. Zaidat, A. Belgacem-Bouzida, L. Ayouni-Derouiche, G. Raimondi, Y. Fautrelle, Int. J. Heat Mass Trans. 55 (2012) 1986.

[17] T. Carozzani, C. Gandin, H. Digonnet, M. Bellet, K. Zaidat, Y. Fautrelle, Met. Mater. Trans. A 44A (2013) 873.

[18] C. Gandin, S. Mosbah, T. Volkmann, D. Herlach, Acta Mater. 56 (2008) 3023.

[19] M. Ahmadein, M. Wu, J. Li, P. Schumacher, A. Ludwig, Met. Mater. Trans. A 44A (2013) 2895.

[20] H. Combeau, M. Založnik, S. Hans, P. Richy, Met. Mater. Trans. B 40B (2009) 289.

[21] J. Li, M. Wu, A. Ludwig, A. Kharicha, in: A. Ludwig (Ed.), MCWASP XIII: International Conference on Modeling of Casting, Welding and Advanced Solidification Processes, IOP Conference Series, vol. 33, 2012.

[22] M. Wu, A. Ludwig, Acta Mater. 57 (2009) 56215631.

[23] M. Wu, M. Ahmadein, A. Kharicha, A. Ludwig, J.H. Li, P. Schumacher, in: A. Ludwig (Ed.), MCWASP XIII: International Conference on Modeling of Casting, Welding and Advanced Solidification Processes, IOP Conference Series, vol. 33, 2012.

[24] H. Diepers, A. Karma, in: M. Rappaz, C. Beckermann, R. Trivedi (Eds.), Solidification Processes and Microstructures: A Symposium in Honor of Wilfried Kurz, TMS Publication, Warrendale, PA, USA, 2004, pp. 369-371.

[25] J. Dantzig, M. Rappaz, Solidification, first ed., EPFL Press, 2009.

[26] C. Gandin, M. Rappaz, Acta Mater. 45 (1997) 2187.

[27] C. Gandin, J. Desbiolles, M. Rappaz, P. Thévoz, Met. Mater. Trans. A 30A (1999) 3153.

[28] T. Carozzani, C. Gandin, H. Digonnet, Modell. Simul. Mater. Sci. Eng. 22 (2014) 015012.

[29] D. Hebditch, J. Hunt, Metall. Trans. 5 (1974) 1557.

[30] G. Reinhart, C. Gandin, N. Mangelinck-Noël, H. Nguyen-Thi, J. Spinelli, J. Baruchel, B. Billia, Acta Mater. 61 (2013) 4765.

[31] D.R. Liu, G. Reinhart, N. Mangelinck-Noël, C. Gandin, H. Nguyen-Thi, B. Billia, ISIJ Int. 54 (2014) 392.

[32] J. Friedli, J. Fife, P.D. Napoli, M. Rappaz, Metall. Mater. Trans. A 44A (2013) 5522.

[33] Y. Zhou, A. Volek, N. Green, Acta Mater. 56 (2008) 2631.

[34] J. Li, Z. Wang, Y. Yang, J. Wang, in: A. Ludwig (Ed.), MCWASP XIII: International Conference on Modeling of Casting, Welding and Advanced Solidification Processes, IOP Conference Series, vol. 33, 2012.

[35] W. Wang, P. Lee, M. McLean, Acta Mater. 51 (2003) 2971.

[36] L. Beltran-Sanchez, D. Stefanescu, Met. Trans. A 34 (2003) 367.

[37] J. Miller, L. Yuan, P. Lee, T. Pollock, Acta Mater. 69 (2014) 4759.

[38] N. Shevchenko, S. Boden, G. Gerbeth, S. Eckert, Met. Mater. Trans. A 44A (2013) 3797.

[39] S. Karagadde, L. Yuan, N. Shevchenko, S. Eckert, P. Lee, Acta Mater. 79 (2014) 168.
[40] L. Tan, N. Zabaras, J. Comput. Phys. 221 (2007) 940.

[41] A. Jacot, M. Rappaz, Acta Mater. 50 (2002) 1909.

[42] I. Steinbach, C. Beckermann, B. Kauerauf, Q. Li, J. Guo, Acta Mater. 47 (1999) 971.

[43] I. Steinbach, H. Diepers, C. Beckermann, J. Cryst. Growth 275 (2005) 624.

[44] P. Delaleau, C. Beckermann, R. Mathiesen, L. Arnberg, ISIJ Int. 50 (2010) 1886.

[45] T. Piwonka, M. Flemings, J. Metals 16 (1964) 762.

[46] K. Kubo, R.D. Pehlke, Met. Trans. B 16B (1985) 359.

[47] P.D. Lee, A. Chirazi, D. See, J. Light Metals 1 (2001) 15.

[48] G. Couturier, M. Rappaz, in: Y.T. Zhu, Q. Wang (Eds.), Simulation of Aluminum Shape Casting Processing, TMS Publication, Warrendale, PA, USA, 2006, p. 143.

[49] K.D. Carlson, Z. Lin, C. Beckermann, Met. Mater. Trans. B 38B (2007) 541.

[50] M. Felberbaum, E. Landry-Desy, L. Weber, M. Rappaz, Acta Mater. 59 (2011) 2302.

[51] M. Felberbaum, M. Rappaz, Acta Mater. 59 (2011) 6849.

[52] D. Kammer, P. Voorhees, Acta Mater. 54 (2006) 1549.

[53] H. Meidani, A. Jacot, J. Desbiolles, M. Rappaz, Acta Mater. 60 (2012) 2518.

[54] H. Meidani, A. Jacot, M. Rappaz, Met. Mater. Trans. A 60 (2014), http:// dx.doi.org/10.1007/s11661.

[55] J.S. Wang, P.D. Lee, Int. J. Cast. Met. Res. 20 (2007) 151.

[56] M. Rappaz, J. Drezet, M. Gremaud, Met. Mater. Trans. A 30A (1999) 449

[57] M. M’Hamdi, A. Mo, H. Fjaer, Met. Mater. Trans. A 37A (2006) 3069.

[58] V. Mathier, J. Drezet, M. Rappaz, Model Simul. Mater. Sci. Eng. 15 (2007) 121.

[59] O. Ludwig, J.M. Drezet, C. Martin, M. Suery, Met. Mater. Trans. A 36A (2005) 1525.

[60] M. Rappaz, A. Jacot, W. Boettinger, Met. Mater. Trans. A 34A (2003) 467.

[61] S.J. Fensin, D. Olmsted, D. Buta, M. Asta, A. Karma, J.J. Hoyt, Phys. Rev. E 81 (2010) 031601.

[62] N. Wang, S. Mokadem, M. Rappaz, W. Kurz, Acta Mater. 52 (2004) 3173.

[63] S. Vernède, P. Jarry, M. Rappaz, Acta Mater. 54 (2006) 4023.

[64] A. Phillion, J. Desbiolles, M. Rappaz, in: S. Cockcroft, D. Maijer (Eds.), Modeling of Casting, Welding and Advanced Solidification Processes XII, TMS Publication, Warrendale, PA, USA, 2009, p. 143.

[65] S. Vernède, J. Dantzig, M. Rappaz, Acta Mater. 57 (2009) 1554

[66] M. Sistaninia, A.B. Phillion, J. Drezet, M. Rappaz, Acta Mater. 60 (2012) 6793.

[67] S. Terzi, L. Salvo, M. Sury, N. Limodin, J. Adrien, E. Maire, Y. Pannier, M. Bornert, D. Bernard, M. Felberbaum, M. Rappaz, E. Boller, Scripta Mater. 61 (2009) 449.

[68] M. Sistaninia, S. Terzi, A.B. Phillion, J. Drezet, M. Rappaz, Acta Mater. 61 (2013) 3831.

[69] J. Zaragoci, L. Silva, M. Bellet, C. Gandin, in: A. Ludwig (Ed.), MCWASP XIII: International Conference on Modeling of Casting, Welding and Advanced Solidification Processes, IOP Conference Series, vol. 33, 2012.

[70] M. Rettenmayr, Int. Mater. Rev. 54 (2009) 1.

[71] M. Rappaz, G. Kurtuldu, Thermodynamic aspects of homogeneous nucleation enhanced by icosahedral short range order in liquid fcc-type alloys, J. Metals 67 (2015) 1812. 